\title{
Nonrelativistic limit of the Dirac-Fock equations.
}

\author{
Maria J. Esteban and Eric Séré \\ CEREMADE (UMR C.N.R.S. 7534) \\ Université Paris IX-Dauphine \\ Place du Maréchal de Lattre de Tassigny \\ 75775 Paris Cedex 16 - France \\ esteban or sere@ceremade.dauphine.fr
}

\begin{abstract}
In this paper, the Hartree-Fock equations are proved to be the non relativistic limit of the Dirac-Fock equations as far as convergence of "stationary states" is concerned. This property is used to derive a meaningful definition of "ground state" energy and "ground state" solutions for the Dirac-Fock model.
\end{abstract}

AMS Subject Classification: 49 S 05, 35 J 60, 35 P 30, 35 Q 75, 81 Q 05, $81 \mathrm{~V} 70,81 \mathrm{~V} 45,81 \mathrm{~V} 55$.

Key Words : Relativistic quantum mechanics, nonrelativistic limit, quantum chemistry, ground state, nonlinear eigenvalue problems, Dirac-Fock equations, Hartree-Fock equations, variational methods, critical points, strongly indefinite functionals, bifurcations from the essential spectrum.

\section{Introduction}

In this paper we prove that solutions of Dirac-Fock equations converge, in a certain sense, towards solutions of the Hartree-Fock equations when the speed of light tends to infinity.

This limiting process allows us to define a notion of ground state for the Dirac-Fock equations, valid when the speed of light is large enough.

First of all, we choose units for which $m=\hbar=1$, where $m$ is the mass of the electron, and $\hbar$ is Planck's constant. We also impose $\frac{e^{2}}{4 \pi \varepsilon_{0}}=1$, with $-e$ the charge of an electron, $\varepsilon_{0}$ the permittivity of the vacuum. 
The Dirac Hamiltonian can be written as

$$
H_{c}=-i c \alpha \cdot \nabla+c^{2} \beta,
$$

where $c>0$ is the speed of light in the above units, $\beta=\left(\begin{array}{cc}\mathbb{I} & 0 \\ 0 & -\mathbb{I}\end{array}\right)$, $\alpha_{k}=\left(\begin{array}{cc}0 & \sigma_{k} \\ \sigma_{k} & 0\end{array}\right) \quad(k=1,2,3)$ and the $\sigma_{k}$ are the well known Pauli matrices. The operator $H_{c}$ acts on 4-spinors, i.e. functions from $\mathbb{R}^{3}$ to $\mathbb{C}^{4}$, and it is self-adjoint in $L^{2}\left(\mathbb{R}^{3}, \mathbb{C}^{4}\right)$, with domain $H^{1}\left(\mathbb{R}^{3}, \mathbb{C}^{4}\right)$ and form-domain $H^{1 / 2}\left(\mathbb{R}^{3}, \mathbb{C}^{4}\right)$. Its spectrum is $\left(-\infty,-c^{2}\right] \cup\left[c^{2},+\infty\right)$.

Let us consider a system of $N$ electrons coupled to a fixed nuclear charge density $e Z \mu$, where $e$ is the charge of the proton, $Z>0$ the total number of protons and $\mu$ is a probability measure defined on $\mathbb{R}^{3}$. Note that in the particular case of $m$ point-like nuclei, each one having atomic number $Z_{i}$ at a fixed location $x_{i}, e Z \mu=\sum_{i=1}^{m} e Z_{i} \delta_{x_{i}}$ and $Z=\sum_{i=1}^{m} Z_{i}$.

In our system of units, the Dirac-Fock equations for such a molecule are given by

$$
\left(\mathrm{DF}_{c}\right)\left\{\begin{array}{c}
\bar{H}_{c, \Psi} \psi_{k}:=H_{c} \psi_{k}-Z\left(\mu * \frac{1}{|x|}\right) \psi_{k}+\left(\rho_{\Psi} * \frac{1}{|x|}\right) \psi_{k} \\
-\int_{\mathbb{R}^{3}} \frac{R_{\Psi}(x, y) \psi_{k}(y)}{|x-y|} d y=\varepsilon_{k}^{c} \psi_{k} \quad(k=1, \ldots N), \\
\operatorname{Gram}_{\mathrm{L}^{2}} \Psi=\mathbb{I}_{\mathrm{N}} \quad\left(\text { i.e } \int_{\mathbb{R}^{3}} \psi_{\mathrm{k}}^{*} \psi_{\mathrm{l}}=\delta_{\mathrm{kl}}, 1 \leq \mathrm{k}, 1 \leq \mathrm{N}\right) .
\end{array}\right.
$$

Here, $\Psi=\left(\psi_{1}, \cdots, \psi_{N}\right)$, each $\psi_{k}$ is a 4 -spinor in $H^{1 / 2}\left(\mathbb{R}^{3}, \mathbb{C}^{4}\right)$ (by bootstrap, $\psi_{k}$ is also in any $W^{1, p}\left(\mathbb{R}^{3}\right)$ space, $\left.1 \leq p<3 / 2\right)$, and

$$
\rho_{\Psi}(x):=\sum_{k=1}^{N} \psi_{k}^{*}(x) \psi_{k}(x), R_{\Psi}(x, y):=\sum_{k=1}^{N} \psi_{k}(x) \otimes \psi_{k}^{*}(y) .
$$

We have denoted $\psi^{*}$ the complex line vector whose components are the conjugates of those of a complex (column) vector $\psi$, and $\psi_{1}^{*} \psi_{2}$ is the inner product of two complex (column) vectors $\psi_{1}, \psi_{2}$. The $n \times n$ matrix $\operatorname{Gram}_{L^{2}} \Psi$ is defined by the usual formulas

$$
\left(\operatorname{Gram}_{\mathrm{L}^{2}} \Psi\right)_{k l}:=\int_{\mathbb{R}^{3}} \psi_{k}^{*}(x) \psi_{l}(x) d x .
$$


Finally, $\varepsilon_{1}^{c} \leq \ldots \leq \varepsilon_{N}^{c}$ are eigenvalues of $\bar{H}_{c, \Psi}$. Each one represents the energy of one of the electrons, in the mean field created by the molecule. For physical reasons, we impose $0<\varepsilon_{k}^{c}<c^{2}$. Note that the scalars $\varepsilon_{k}^{c}$ can also be seen as Lagrange multipliers. Indeed, the Dirac-Fock equations are the Euler-Lagrange equations of the Dirac-Fock energy functional

$$
\begin{aligned}
\mathcal{E}_{c}(\Psi)=\sum_{k=1}^{N} & \int_{\mathbb{R}^{3}} \psi_{k}^{*} H_{c} \psi_{k}-Z\left(\mu * \frac{1}{|x|}\right) \psi_{k}^{*} \psi_{k} \\
& +\frac{1}{2} \iint_{\mathbb{R}^{3} \times \mathbb{R}^{3}} \frac{\rho_{\Psi}(x) \rho_{\Psi}(y)-\operatorname{tr}\left(R_{\Psi}(x, y) R_{\Psi}(y, x)\right)}{|x-y|} d x d y
\end{aligned}
$$

under the constraints $\int_{\mathbb{R}^{3}} \psi_{k}^{*} \psi_{l}=\delta_{k l}$.

In [6] we proved that under some assumptions on $N$ and $Z$, there exists an infinite sequence of solutions of $\left(\mathrm{DF}_{c}\right)$. More precisely:

Theorem 1 [6]. Let $N<Z+1$. For any $c>\frac{\pi / 2+2 / \pi}{2} \max (Z, 3 N-1)$, there exists a sequence of solutions of $\left(D F_{c}\right),\left\{\Psi^{c, j}\right\}_{j \geq 1} \subset\left(H^{1 / 2}\left(\mathbb{R}^{3}\right)\right)^{N}$, such that

(i) $0<\mathcal{E}_{c}\left(\Psi^{c, j}\right)<N c^{2}$,

(ii) $\lim _{j \rightarrow+\infty} \mathcal{E}_{c}\left(\Psi^{c, j}\right)=N c^{2}$,

(iii) $0<c^{2}-\mu_{j}<\varepsilon_{1}^{c, j} \leq \ldots \leq \varepsilon_{N}^{c, j}<c^{2}-m_{j}$, with $\mu_{j}>m_{j}>0$ independent of $c$.

The constant $\frac{\pi / 2+2 / \pi}{2}$ is related to a Hardy-type inequality obtained independently by Tix and Burenkov-Evans (see [15, 3, 16]), and which plays an important role in the proof of Theorem 1 . With the physical value $c=$ $137.037 \ldots$ and $Z$ an integer (the total number of protons in the molecule), our conditions become $N \leq Z, N \leq 41, Z \leq 124$. The constraint $N \leq 41$ is technical, and has no physical meaning.

Our result was recently improved by Paturel [13], who relaxed the condition on $N$. Paturel obtains the same multiplicity result, assuming only that $N<Z+1$ and $\frac{\pi / 2+2 / \pi}{2} \max (Z, N)<c$. Taking $c=137.037 \ldots$, Paturel's conditions are $N \leq Z \leq 124$ : they cover all existing neutral atoms. This is an important improvement. 
In [6], the critical points $\Psi^{c, j}$ are obtained by a complicated min-max argument (in [13], the argument is not simpler). Here, we do not give this min-max in its full detail. We just state the minimal information needed in the present paper. Let us denote $E:=H^{1 / 2}\left(\mathbb{R}^{3}, \mathbb{C}^{4}\right)$. Since

$$
\sigma\left(H_{c}\right)=\left(-\infty,-c^{2}\right] \cup\left[c^{2},+\infty\right)
$$

the Hilbert space $E$ can be split as

$$
E=E_{c}^{+} \oplus E_{c}^{-}
$$

where $E_{c}^{ \pm}:=\Lambda_{c}^{ \pm} E$, and $\Lambda_{c}^{ \pm}:=\chi_{\mathbb{R}_{ \pm}}\left(H_{c}\right)$. The projectors $\Lambda_{c}^{ \pm}$have a simple expression in the Fourier domain : $\widehat{\Lambda_{c}^{ \pm}} \psi(\xi)=\hat{\Lambda}_{c}^{ \pm}(\xi) \hat{\psi}(\xi)$, with

$$
\widehat{\Lambda}_{c}^{ \pm}(\xi):=\frac{1}{2}\left(\mathbb{I}_{\mathbb{d}^{4}} \pm \frac{\mathrm{c} \alpha \cdot \xi+\mathrm{c}^{2} \beta}{\sqrt{\mathrm{c}^{4}+\mathrm{c}^{2}|\xi|^{2}}}\right) .
$$

Proposition $2[\mathbf{6}, \mathbf{1 3}]$. For every $j \geq 1$, let $V$ be any $(N+j-1)$ dimensional complex subspace of $E_{c}^{+}$. Then, taking the notation of Theorem 1 , we have

$$
E_{j, D F}^{c}:=\mathcal{E}_{c}\left(\Psi^{c, j}\right) \leq \sup _{\substack{\Psi \in\left(E_{c}^{-} \oplus V\right)^{N} \\ \operatorname{Gram}_{\mathrm{L}^{2}} \Psi \leq \mathbb{I}_{\mathrm{N}}}} \mathcal{E}_{c}(\Psi) .
$$

In the present paper, we prove three main theorems. We first consider a sequence $c_{n} \rightarrow+\infty$ and a sequence $\left\{\Psi^{n}\right\}_{n}$ of solutions of $\left(\mathrm{DF}_{c_{n}}\right)$. For all $n, \Psi^{n}=\left(\psi_{1}^{n}, \ldots, \psi_{N}^{n}\right)$, each $\psi_{k}^{n}$ is in $H^{1 / 2}\left(\mathbb{R}^{3}, \mathbb{C}^{4}\right)$, with $\int_{\mathbb{R}^{3}} \psi_{k}^{*} \psi_{l} d x=\delta_{k l}$ and $\bar{H}_{c_{n}, \Psi^{n}} \psi_{k}^{n}=\varepsilon_{k}^{n} \psi_{k}^{n}$. Using the standard Hardy inequality, one can prove that the functions $\psi_{k}^{n}$ are in $H^{1}\left(\mathbb{R}^{3}, \mathbb{C}^{4}\right)$ for $c_{n}$ large enough. We assume that

$$
-\infty<\underset{n \rightarrow+\infty}{\varliminf_{\rightarrow}}\left(\varepsilon_{1}^{n}-c_{n}^{2}\right) \leq \varlimsup_{n \rightarrow+\infty}\left(\varepsilon_{N}^{n}-c_{n}^{2}\right)<0 .
$$

A (column) vector $\psi \in \mathbb{C}^{4}$ can be written in block form $\psi=\left(\begin{array}{l}\varphi \\ \chi\end{array}\right)$ where $\varphi \in \mathbb{C}^{2}$ (respectively $\chi \in \mathbb{C}^{2}$ ) consists of the two upper (resp. lower) components of $\psi$. This gives the splitting $\psi_{k}^{n}=\left(\begin{array}{l}\varphi_{k}^{n} \\ \chi_{k}^{n}\end{array}\right)$ with $\varphi_{k}^{n}$ and $\chi_{k}^{n}$ in $H^{1}\left(\mathbb{R}^{3}, \mathbb{C}^{2}\right)$. Finally, $\Psi^{n}$ splits as $\left(\begin{array}{c}\Phi^{n} \\ \chi^{n}\end{array}\right)$, where $\Phi^{n}:=\left(\varphi_{1}^{n}, \ldots, \varphi_{N}^{n}\right)$ and $\chi^{n}:=\left(\chi_{1}^{n}, \ldots, \chi_{N}^{n}\right)$. Our first result is that $\Psi^{n}=\left(\begin{array}{c}\Phi^{n} \\ \chi^{n}\end{array}\right)$ has a subsequence converging, in $H^{1}$ norm, 
towards $\bar{\Psi}=\left(\begin{array}{c}\bar{\Phi} \\ 0\end{array}\right)$, where $\bar{\Phi}=\left(\bar{\varphi}_{1}, \cdots, \bar{\varphi}_{N}\right) \in\left(H^{1}\left(\mathbb{R}^{3}, \mathbb{C}^{2}\right)\right)^{N}$ is a solution of the Hartree-Fock equations:

$$
\left\{\begin{array}{c}
\mathcal{H}_{\Phi} \varphi_{k}=-\frac{\Delta \varphi_{k}}{2}-Z\left(\mu * \frac{1}{|x|}\right) \varphi_{k}+\left(\rho_{\Phi} * \frac{1}{|x|}\right) \varphi_{k} \\
-\int_{\mathbb{R}^{3}} \frac{R_{\Phi}(x, y) \varphi_{k}(y)}{|x-y|} d y=\bar{\lambda}_{k} \varphi_{k}, \quad k=1, \ldots N, \\
\int_{\mathbb{R}^{3}} \varphi_{k}^{*} \varphi_{l} d x=\delta_{k l}, \quad \bar{\lambda}_{k}=\lim _{n \rightarrow+\infty}\left(\varepsilon_{k}^{n}-c_{n}^{2}\right) .
\end{array}\right.
$$

Here (as in the Dirac-Fock equations),

$$
\rho_{\Phi}(x)=\sum_{l=1}^{N} \varphi_{l}^{*}(x) \varphi_{l}(x), \quad R_{\Phi}(x, y)=\sum_{l=1}^{N} \varphi_{l}(x) \otimes \varphi_{l}^{*}(y) .
$$

Note that the Hartree-Fock equations are the Euler-Lagrange equations corresponding to critical points in $\left(H^{1}\left(\mathbb{R}^{3}, \mathbb{C}^{2}\right)\right)^{N}$ of the Hartree-Fock energy:

$$
\mathcal{E}_{H F}(\Phi):=\sum_{k=1}^{N} \frac{1}{2}\left\|\nabla \varphi_{k}\right\|_{L^{2}}^{2}-Z \int_{\mathbb{R}^{3}}\left(\mu * \frac{1}{|x|}\right)\left|\varphi_{k}\right|^{2} d x
$$

$$
+\frac{1}{2} \iint_{\mathbb{R}^{3} \times \mathbb{R}^{3}} \frac{\rho_{\Phi}(x) \rho_{\Phi}(y)-\operatorname{tr}\left(R_{\Phi}(x, y) R_{\Phi}(y, x)\right)}{|x-y|} d x d y
$$

under the constraint $\int_{\mathbb{R}^{3}} \varphi_{k}^{*} \varphi_{l}=\delta_{k l}, i, j=1, \ldots N$.

Theorem 3 . Let $N<Z+1$. Consider a sequence $c_{n} \rightarrow+\infty$ and $a$ sequence $\left\{\Psi^{n}\right\}_{n}$ of solutions of $\left(D F_{c_{n}}\right)$, i.e. $\Psi^{n}=\left(\psi_{1}^{n}, \cdots, \psi_{N}^{n}\right)$, each $\psi_{k}^{n}$ being in $H^{1 / 2}\left(\mathbb{R}^{3}, \mathbb{C}^{4}\right)$, with $\int_{\mathbb{R}^{3}} \psi_{k}^{*} \psi_{l} d x=\delta_{k l}$ and $\bar{H}_{c_{n}, \Psi^{n}} \psi_{k}^{n}=\varepsilon_{k}^{n} \psi_{k}^{n}$. Assume that the multipliers $\varepsilon_{k}^{n}, k=1, \ldots, N$, satisfy (6). Then for $n$ large enough, $\psi_{k}^{n}$ is in $H^{1}\left(\mathbb{R}^{3}, \mathbb{C}^{4}\right)$, and there exists a solution of $(H F)$, $\bar{\Phi}=\left(\bar{\varphi}_{1}, \cdots, \bar{\varphi}_{N}\right)$, with negative multipliers, $\bar{\lambda}_{1}, \ldots, \bar{\lambda}_{N}$, such that, after extraction of a subsequence,

$$
\lambda_{k}^{n}:=\varepsilon_{k}^{n}-\left(c_{n}\right)^{2} \underset{n \rightarrow+\infty}{\longrightarrow} \bar{\lambda}_{k}, \quad k=1, \ldots, N,
$$




$$
\begin{gathered}
\psi_{k}^{n}=\left(\begin{array}{c}
\varphi_{k}^{n} \\
\chi_{k}^{n}
\end{array}\right) \underset{n \rightarrow+\infty}{\longrightarrow}\left(\begin{array}{c}
\bar{\varphi}_{k} \\
0
\end{array}\right) \text { in } H^{1}\left(\mathbb{R}^{3}, \mathbb{C}^{2}\right) \times H^{1}\left(\mathbb{R}^{3}, \mathbb{C}^{2}\right), \\
\left\|\chi_{k}^{n}+\frac{i}{2 c_{n}}(\sigma \cdot \nabla) \varphi_{k}^{n}\right\|_{L^{2}\left(\mathbb{R}^{3}, \mathbb{C}^{2}\right)}=O\left(1 /\left(c_{n}\right)^{3}\right),
\end{gathered}
$$

and

$$
\mathcal{E}_{c_{n}}\left(\Psi^{n}\right)-N c_{n}^{2} \underset{n \rightarrow+\infty}{\longrightarrow} \quad \mathcal{E}_{H F}(\bar{\Phi}) .
$$

As a particular case, we have

Corollary 4 . If $c_{n} \rightarrow+\infty$ and $N, Z, \mu$ are fixed, then for any $j \geq 1$ the sequence $\left\{\Psi^{c_{n}, j}\right\}_{n}$ of Theorem 1 satisfies the assumptions of Theorem 3 (see (iii) in Theorem 1). So it is precompact in $\left(H^{1}\left(\mathbb{R}^{3}, \mathbb{C}^{4}\right)\right)^{N}$. Up to extraction of subsequences,

$$
\begin{gathered}
\lambda_{k}^{c_{n}, j}:=\varepsilon_{k}^{c_{n}, j}-c_{n}^{2} \longrightarrow \bar{\lambda}_{k}^{j}<0, k=1, \ldots, N \\
\Psi^{c_{n}, j} \longrightarrow\left(\begin{array}{c}
\bar{\Phi}^{j} \\
0
\end{array}\right) \quad \text { in }\left(H^{1}\left(\mathbb{R}^{3}, \mathbb{C}^{2}\right)\right)^{N} \times\left(H^{1}\left(\mathbb{R}^{3}, \mathbb{C}^{2}\right)\right)^{N}
\end{gathered}
$$

and $\bar{\Phi}^{j}=\left(\bar{\varphi}_{1}^{j}, \cdots, \bar{\varphi}_{n}^{j}\right)$ is a solution of the Hartree-Fock equations with multipliers $\bar{\lambda}_{1}^{j}, \cdots, \bar{\lambda}_{N}^{j}$. Moreover,

$$
\mathcal{E}_{c_{n}}\left(\Psi^{c_{n}, j}\right)-N c_{n}^{2} \underset{n \rightarrow+\infty}{\longrightarrow} \quad \mathcal{E}_{H F}\left(\bar{\Phi}^{j}\right) .
$$

Particular solutions of the Hartree-Fock equations are the minimizers of $\mathcal{E}_{H F}(\Phi)$ under the constraints $\operatorname{Gram}_{\mathrm{L}^{2}} \Phi=\mathbf{I}_{\mathrm{N}}$. They are called ground states. Their existence was proved by Lieb and Simon [10] under the assumption $N<Z+1$ (see also [11] for the existence of excited states).

It is difficult to define the notion of ground state for the Dirac-Fock model, since $\mathcal{E}_{c}$ has no minimum under the constraints $\int_{\mathbb{R}^{3}} \psi_{k}^{*} \psi_{l}=\delta_{k l}$. Our second main result asserts that the first solution $\Psi^{c, 1}$ of $\left(\mathrm{DF}_{c}\right)$ found in [6], whose energy level will be denoted $E_{1, D F}^{c}$, can be considered, in some (weak) sense, as a ground state for $\left(\mathrm{DF}_{c}\right)$. Indeed, $E_{1, D F}^{c}-N c^{2}$ converges to the minimum of $\mathcal{E}_{H F}$ as $c$ goes to infinity. Moreover, for $c$ large the multipliers $\varepsilon_{k}^{c, 1}$ associated to $\Psi^{c, 1}$ are the $N$ smallest positive eigenvalues of the mean-field operator $\bar{H}_{c, \Psi c, 1}$. 
Theorem 5. Let $N<Z+1$ and $c$ sufficiently large. With the above notations,

$$
E_{1, D F}^{c}:=\mathcal{E}_{c}\left(\Psi^{c, 1}\right)=\min _{\operatorname{Gram}_{\mathrm{L}^{2}} \Phi=\mathbf{I}_{\mathrm{N}}} \mathcal{E}_{H F}(\Phi)+N c^{2}+o(1)_{c \rightarrow+\infty}
$$

Moreover, for any subsequence $\left\{\Psi^{c_{n}, 1}\right\}_{n}$ converging in $\left(H^{1}\left(\mathbb{R}^{3}, \mathbb{C}^{4}\right)\right)^{N}$ to some $\left(\begin{array}{c}\bar{\Phi}^{1} \\ 0\end{array}\right), \bar{\Phi}^{1}$ is a ground state of the Hartree-Fock model, i.e.

$$
\mathcal{E}_{H F}\left(\bar{\Phi}^{1}\right)=\min _{\operatorname{Gram}_{\mathrm{L}^{2}} \Phi=\mathbf{I}_{\mathrm{N}}} \mathcal{E}_{H F}(\Phi) .
$$

Furthermore, for c large, the eigenvalues corresponding to $\Psi^{c, 1}$ in $\left(D F_{c}\right)$, $\varepsilon_{1}^{c, 1}, \ldots, \varepsilon_{N}^{c, 1}$ are the smallest positive eigenvalues of the linear operator $\bar{H}_{c, \Psi^{c, 1}}$ and the $(N+1)$-th positive eigenvalue of this operator is strictly larger than $\varepsilon_{N}^{c, 1}$.

Finally, we are able to show that, for $c$ large enough, the function $\Psi^{c, 1}$ can be viewed as an electronic ground state for the Dirac-Fock equations in the following sense: it minimizes the Dirac-Fock energy among all electronic configurations which are orthogonal to the "Dirac sea".

Theorem 6 . Fix $N, Z$ with $N<Z+1$ and take $c$ sufficiently large. Then $\Psi^{c, 1}$ is a solution of the following minimization problem:

$$
\inf \left\{\mathcal{E}_{c}(\Psi) ; \operatorname{Gram}_{\mathrm{L}^{2}} \Psi=\mathbf{I}_{\mathrm{N}}, \Lambda_{\Psi}^{-} \Psi=0\right\}
$$

where $\Lambda_{\Psi}^{-}=\chi_{(-\infty, 0)}\left(\bar{H}_{c, \Psi}\right)$ is the negative spectral projector of the operator $\bar{H}_{c, \Psi}$, and $\Lambda_{\Psi}^{-} \Psi:=\left(\Lambda_{\Psi}^{-} \psi_{1}, \cdots, \Lambda_{\Psi}^{-} \psi_{N}\right)$.

The constraint $\Lambda_{\Psi}^{-} \Psi=0$ has a physical meaning. Indeed, according to Dirac's original ideas, the vacuum consists of infinitely many electrons which completely fill up the negative space of $\bar{H}_{c, \Psi}$ : these electrons form the "Dirac sea". So, by the Pauli exclusion principle, additional electronic states should be in the positive space of the mean-field Hamiltonian $\bar{H}_{c, \Psi}$. The proof of Theorem 6 will be given in Section 4. This proof uses some other interesting min-max characterizations of $\Psi^{c, 1}$ (see Lemma 9 ). 


\section{The nonrelativistic limit}

This section is devoted to the proof of Theorem 3 . We first notice that when $N<Z+1, N, Z$ fixed, and $c$ is sufficiently large, any solution of ( $\left.\mathrm{DF}_{c}\right)$ is actually in $\left(H^{1}\left(\mathbb{R}^{3}\right)\right)^{N}$. This follows from the fact that for $\nu$ small, the operator $H_{1}-\frac{\nu}{|x|}$ is essentially self-adjoint with domain $H^{1}\left(\mathbb{R}^{3}\right)$ (see [14]).

We can also obtain a priori estimates on $H^{1}$ norms:

Lemma 7 . Fix $N, Z \in \mathbb{Z}^{+}$, take c large enough, and let $\Psi^{c}$ be a solution of $\left(D F_{c}\right)$. If the multipliers $\varepsilon_{k}^{c}$ associated to $\Psi^{c}$ satisfy $0 \leq \varepsilon_{k}^{c} \leq c^{2}$ $(k=1, \ldots, N)$, then $\Psi^{c} \in\left(H^{1}\left(\mathbb{R}^{3}, \mathbb{C}^{4}\right)\right)^{N}$, and the following estimate holds

$$
\left\|\Psi^{c}\right\|_{2}^{2}+\left\|\nabla \Psi^{c}\right\|_{2}^{2} \leq K
$$

The constant $K$ is independent of $c$ (for c large).

Proof. The normalization constraint $\operatorname{Gram}_{L^{2}} \Psi^{c}=\mathbb{I}_{\mathrm{N}}$ implies

$$
\left\|\Psi^{c}\right\|_{2}^{2}=N
$$

Using the $\left(\mathrm{DF}_{c}\right)$ equation and the standard Hardy inequality

$$
\int_{\mathbb{R}^{3}} \frac{u^{2}}{|x|^{2}} \leq 4 \int_{\mathbb{R}^{3}}|\nabla u|^{2}
$$

one easily proves that $\Psi^{c}$ is in $H^{1}$, and satisfies:

$$
\begin{gathered}
\left(H_{c} \Psi^{c}, H_{c} \Psi^{c}\right)=c^{4}\left\|\Psi^{c}\right\|_{2}^{2}+c^{2}\left\|\nabla \Psi^{c}\right\|_{2}^{2} \\
\leq c^{4}\left\|\Psi^{c}\right\|_{2}^{2}+\ell\left(Z^{2}+N^{2}\right)\left\|\nabla \Psi^{c}\right\|_{2}^{2}+\ell c^{2} \max (N, Z)\left\|\nabla \Psi^{c}\right\|_{2},
\end{gathered}
$$

for some $\ell>0$ independent of $N, Z$ and $c$. The estimates (18) and (20) prove the lemma.

Proof of Theorem 3. Let us split the spinors $\psi_{k}^{n}: \mathbb{R}^{3} \rightarrow \mathbb{C}^{4}$ in blocks of upper and lower components : $\psi_{k}^{n}=\left(\begin{array}{c}\varphi_{k}^{n} \\ \chi_{k}^{n}\end{array}\right)$, with $\varphi_{k}^{n}, \chi_{k}^{n}: \mathbb{R}^{3} \rightarrow \mathbb{C}^{2}$. We 
denote $L:=-i(\sigma \cdot \nabla)$. Then we can rewrite $\left(\mathrm{DF}_{c_{n}}\right)$ in the following way:

$$
(21)\left\{\begin{array}{l}
c_{n} L \chi_{k}^{n}-Z\left(\mu * \frac{1}{|x|}\right) \varphi_{k}^{n}+\left(\sum_{l=1}^{N}\left(\left|\varphi_{l}^{n}\right|^{2}+\left|\chi_{l}^{n}\right|^{2}\right) * \frac{1}{|x|}\right) \varphi_{k}^{n}+\left(c_{n}\right)^{2} \varphi_{k}^{n} \\
-\sum_{l=1}^{N} \varphi_{l}^{n}(x) \int_{\mathbb{R}^{3}} \frac{\left(\varphi_{l}^{n}\right)^{*}(y) \varphi_{k}^{n}(y)+\left(\chi_{l}^{n}\right)^{*}(y) \chi_{k}^{n}(y)}{|x-y|} d y=\varepsilon_{k}^{n} \varphi_{k}^{n} \\
c_{n} L \varphi_{k}^{n}-Z\left(\mu * \frac{1}{|x|}\right) \chi_{k}^{n}+\left(\sum_{l=1}^{N}\left(\left|\varphi_{l}^{n}\right|^{2}+\left|\chi_{l}^{n}\right|^{2}\right) * \frac{1}{|x|}\right) \chi_{k}^{n}-\left(c_{n}\right)^{2} \chi_{k}^{n} \\
-\sum_{l=1}^{N} \chi_{l}^{n}(x) \int_{\mathbb{R}^{3}} \frac{\left(\varphi_{l}^{n}\right)^{*}(y) \varphi_{k}^{c}(y)+\left(\chi_{l}^{c}\right)^{*}(y) \chi_{k}^{c}(y)}{|x-y|} d y=\varepsilon_{k}^{n} \chi_{k}^{n} \\
\int_{\mathbb{R}^{3}}\left(\varphi_{k}^{n}\right)^{*} \varphi_{l}^{n}+\left(\chi_{l}^{n}\right)^{*} \chi_{l}^{n} d x=\delta_{k l}
\end{array}\right.
$$

Note that $\|L \chi\|_{L^{2}}=\|\nabla \chi\|_{L^{2}}$ for all $\chi \in H^{1}\left(\mathbb{R}^{3}, \mathbb{C}^{2}\right)$. So, dividing by $c_{n}$ the first equation of (21), we get

$$
\left\|\nabla \chi_{k}^{n}\right\|_{L^{2}\left(\mathbb{R}^{3}, \mathbb{C}^{2}\right)}=O\left(1 / c_{n}\right) .
$$

Dividing by $2\left(c_{n}\right)^{2}$ the second equation of (21), and using the fact that $\varepsilon_{k}^{n}-\left(c_{n}\right)^{2}$ is a bounded sequence, we get

$$
\left\|\chi_{k}^{n}-\frac{1}{2 c_{n}} L \varphi_{k}^{n}\right\|_{L^{2}\left(\mathbb{R}^{3}, \mathbb{C}^{2}\right)}=\frac{1}{\left(c_{n}\right)^{2}} O\left(\sum_{l=1}^{N}\left\|\chi_{l}^{n}\right\|_{H^{1}\left(\mathbb{R}^{3}, \mathbb{C}^{2}\right)}\right) .
$$

The estimate (23) together with Lemma 7 implies

$$
\left\|\chi_{k}^{n}\right\|_{L^{2}\left(\mathbb{R}^{3}, \mathbb{d}^{2}\right)}=O\left(1 / c_{n}\right) .
$$

Combining this with (22), we obtain

So $\sum_{l=1}^{N}\left\|\chi_{l}^{n}\right\|_{H^{1}\left(\mathbb{R}^{3}, \mathbb{C}^{2}\right)}=O\left(1 / c_{n}\right)$, and (23) gives the estimate

$$
\left\|\chi_{k}^{n}-\frac{1}{2 c_{n}} L \varphi_{k}^{n}\right\|_{L^{2}\left(\mathbb{R}^{3}, \mathbb{C}^{2}\right)}=O\left(1 /\left(c_{n}\right)^{3}\right)
$$


Now, the first equation of (21), combined with (26), implies

$$
\left\{\begin{array}{l}
-\frac{\Delta \varphi_{k}^{n}}{2}-Z\left(\mu * \frac{1}{|x|}\right) \varphi_{k}^{n}+\left(\sum_{l=1}^{N}\left|\varphi_{l}^{n}\right|^{2} * \frac{1}{|x|}\right) \chi_{k}^{n} \\
\quad-\sum_{l=1}^{N} \varphi_{l}^{n}(x) \int_{\mathbb{R}^{3}} \frac{\left(\varphi_{l}^{n}\right)^{*}(y) \varphi_{k}^{n}(y)}{|x-y|} d y=\lambda_{k}^{n} \varphi_{k}^{n}+h_{k}^{n}, \\
\int_{\mathbb{R}^{3}}\left(\varphi_{k}^{n}\right)^{*} \varphi_{l}^{n}=\delta_{k l}+r_{k l}^{n},
\end{array}\right.
$$

with $\lambda_{k}^{n}:=\varepsilon_{k}^{n}-\left(c_{n}\right)^{2}$, and

$$
\left.\lim _{n \rightarrow+\infty}|| h_{k}^{n}\right|_{H^{-1}\left(\mathbb{R}^{3}\right)}=0, \quad \lim _{n \rightarrow+\infty}\left|r_{k l}^{n}\right|=0 \text { for all } k, l \in\{1, \ldots, N\} .
$$

Therefore $\Phi^{n}:=\left(\varphi_{1}^{n}, \ldots, \varphi_{N}^{n}\right)$ is a Palais-Smale sequence for the HartreeFock problem, and the multipliers $\lambda_{k}^{n}$ satisfy $\varlimsup_{n \rightarrow+\infty} \lambda_{k}^{n}<0$. At this point, we just invoke an argument used in [11] to obtain the convergence in $H^{1}$ norm of some subsequence $\left\{\Phi^{n^{\prime}}\right\}$ towards $\bar{\Phi}=\left(\bar{\varphi}_{1}, \cdots, \bar{\varphi}_{N}\right)$, a solution of the Hartree-Fock equations

$$
\left\{\begin{array}{l}
\mathcal{H}_{\bar{\Phi}} \bar{\varphi}_{k}=\bar{\lambda}_{k} \bar{\varphi}_{k}, \quad k=1, \ldots N \\
\int_{\mathbb{R}^{3}} \bar{\varphi}_{k}^{*} \bar{\varphi}_{l}=\delta_{k l},
\end{array}\right.
$$

where $\bar{\lambda}_{k}=\lim _{n^{\prime} \rightarrow+\infty} \lambda_{k}^{n^{\prime}}$.

Finally, let us prove that $\mathcal{E}_{c_{n^{\prime}}}\left(\Psi^{n^{\prime}}\right)-N\left(c_{n^{\prime}}\right)^{2}$ converges to $\mathcal{E}_{H F}(\bar{\Phi})$. From Lemma 7 and the estimate $(26)$, one easily gets

$$
\mathcal{E}_{c_{n}}\left(\Psi^{n}\right)-N c_{n}^{2}=\mathcal{E}_{H F}\left(\Phi^{n}\right)+O\left(1 /\left(c_{n}\right)^{2}\right) .
$$

Since $\Phi^{n^{\prime}}$ converges in $H^{1}$ norm to $\bar{\Phi}$, the energy level $\mathcal{E}_{H F}\left(\Phi^{n^{\prime}}\right)$ converges to $\mathcal{E}_{H F}(\bar{\Phi})$. So $(27)$ implies the desired convergence. This ends the proof of Theorem 3 . 


\section{Ground state for Dirac-Fock equations in the nonrelativistic limit.}

The aim of this section is to prove Theorem 5 . The estimate given in Proposition 2 on the energy $\mathcal{E}_{c}\left(\Psi^{c, i}\right)$ and the expression of $\hat{\Lambda}_{c}^{ \pm}$given in (4), will be crucial.

Proof of Theorem 5. By Corollary 4, for any sequence $c_{n}$ going to infinity, $\Psi^{c_{n}, 1}$ is precompact in $H^{1}$ norm. If it converges, its limit is of the form $\left(\begin{array}{c}\bar{\Phi}^{1} \\ 0\end{array}\right)$, and $\left(\mathcal{E}_{c_{n}}\left(\Psi^{c_{n}, 1}\right)-N\left(c_{n}\right)^{2}\right)$ converges to $\mathcal{E}_{H F}\left(\bar{\Phi}^{1}\right)$. As a consequence,

$$
\varliminf_{c \rightarrow+\infty}\left(\mathcal{E}_{c}\left(\Psi^{c, 1}\right)-N c^{2}\right) \geq \inf _{\operatorname{Gram}_{\mathrm{L}^{2}} \Phi=\mathbf{I}_{\mathrm{N}}} \mathcal{E}_{H F}(\Phi) .
$$

In order to prove (15) and (16) of Theorem 5, we just have to show that

$$
\varlimsup_{c \rightarrow+\infty}\left(\mathcal{E}_{c}\left(\Psi^{c, 1}\right)-N c^{2}\right) \leq \inf _{\operatorname{Gram}_{\mathrm{L}^{2}} \Phi=\mathbf{I}_{\mathrm{N}}} \mathcal{E}_{H F}(\Phi) .
$$

Take $\Phi=\left(\varphi_{1}, \cdots, \varphi_{N}\right) \in\left(H^{1}\left(\mathbb{R}^{3}, \mathbb{C}^{2}\right)\right)^{N}$, with $\operatorname{Gram}_{L^{2}} \Phi=\mathbb{I}_{\mathrm{N}}$. Let $V_{c}$ be the complex subspace of $E_{c}^{+}$defined by

$$
V_{c}:=\operatorname{Span}\left\{\Lambda_{c}^{+}\left(\begin{array}{c}
\varphi_{1} \\
0
\end{array}\right), \ldots, \Lambda_{c}^{+}\left(\begin{array}{c}
\varphi_{N} \\
0
\end{array}\right)\right\}
$$

From formula (4) and Lebesgue's convergence theorem, one easily gets, for $k=1, \ldots, N$,

$$
\lim _{c \rightarrow+\infty}\left\|\Lambda_{c}^{-}\left(\begin{array}{c}
\varphi_{k} \\
0
\end{array}\right)\right\|_{H^{1}}=0 .
$$

So, for $c$ sufficiently large, we have

$$
\operatorname{dim} V_{c}=N
$$

Hence, by (5),

$$
E_{1, D F}^{c}:=\mathcal{E}_{c}\left(\Psi^{c, 1}\right) \leq \sup _{\substack{\Psi \in\left(E^{-} \oplus V_{c}\right)^{N} \\ \operatorname{Gram}_{\mathrm{L}^{2}} \Psi \leq \mathbf{I}_{\mathrm{N}}}} \mathcal{E}_{c}(\Psi) .
$$

Let $\Psi^{+} \in\left(E_{c}^{+}\right)^{N}, \Psi^{-} \in\left(E_{c}^{-}\right)^{N}$ such that $\operatorname{Gram}_{L^{2}}\left(\Psi^{+}+\Psi^{-}\right) \leq \mathbb{1}_{\mathrm{N}}$. By the concavity property of $\mathcal{E}_{c}$ in the $E_{c}^{-}$direction (see [6], Lemma 2.2), if $c$ 
is large enough, we have

$$
\begin{aligned}
\mathcal{E}_{c}\left(\Psi^{+}+\Psi^{-}\right) & \leq \mathcal{E}_{c}\left(\Psi^{+}\right)+\mathcal{E}_{c}^{\prime}\left(\Psi^{+}\right) \cdot \Psi^{-}-\frac{1}{4} \sum_{k=1}^{N}\left(\psi_{k}^{-}, \sqrt{-c^{2} \Delta+c^{4}} \psi_{k}^{-}\right) \\
& \leq \mathcal{E}_{c}\left(\Psi^{+}\right)+M\left\|\Psi^{-}\right\|_{L^{2}}-\frac{c^{2}}{4}\left\|\Psi^{-}\right\|_{L^{2}}^{2},
\end{aligned}
$$

for some constant $M>0$ independent of $c$. Hence, for $c$ large,

$$
E_{1, D F}^{c} \leq \sup _{\Psi^{+} \in D\left(V_{c}\right)} \mathcal{E}_{c}\left(\Psi^{+}\right)+\circ(1)_{c \rightarrow+\infty}
$$

where $D\left(V_{c}\right):=\left\{\Psi^{+} \in\left(V_{c}\right)^{N} ; \operatorname{Gram}_{\mathrm{L}^{2}} \Psi^{+} \leq \mathbf{I}_{\mathrm{N}}\right\}$.

If $c$ is large enough, it follows from Hardy's inequality (19) that the map $\Psi^{+} \rightarrow \mathcal{E}_{c}\left(\Psi^{+}\right)$is strictly convex on the convex set

$$
\mathcal{A}^{+}:=\left\{\Psi^{+} \in\left(E_{c}^{+}\right)^{N} ; \operatorname{Gram}_{\mathrm{L}^{2}} \Psi^{+} \leq \mathbb{I}_{\mathrm{N}}\right\} .
$$

Indeed, its second derivative at any point $\Psi^{+}$of $\mathcal{A}^{+}$is of the form

$$
\mathcal{E}_{c}^{\prime \prime}\left(\Psi^{+}\right)\left[d \Psi^{+}\right]^{2}=2 \sum_{k=1}^{N}\left(d \psi_{k}, \sqrt{c^{4}-c^{2} \Delta} d \psi_{k}\right)_{L^{2}}+Q\left(d \Psi^{+}\right)
$$

with $Q$ a quadratic form on $\left(H^{1 / 2}\left(\mathbb{R}^{3}, \mathbb{C}^{4}\right)\right)^{N}$ bounded independently of $c$ and $\Psi^{+} \in \mathcal{A}^{+}$.

As a consequence, $\sup _{\Psi^{+} \in D\left(V_{c}\right)} \mathcal{E}_{c}\left(\Psi^{+}\right)$is achieved by an extremal point $\Psi_{\max }^{+}$ of the convex set $D\left(V_{c}\right)=\mathcal{A}^{+} \cap\left(V_{c}\right)^{N}$. Being extremal in $D\left(V_{c}\right)$, the point $\Psi_{\max }^{+}$satisfies

$$
\operatorname{Gram}_{\mathrm{L}^{2}} \Psi_{\max }^{+}=\mathbf{I}_{\mathrm{N}} .
$$

Since $\psi_{k, \text { max }}^{+} \in V_{c}$, there is a matrix $A=\left(a_{k l}\right)_{1 \leq k, l \leq N}$ such that, for all $l$, $\psi_{l, \max }^{+}=\sum_{1 \leq k \leq N} a_{k l} \Lambda_{c}^{+}\left(\begin{array}{c}\varphi_{k} \\ 0\end{array}\right)$. Then

$$
A^{*} \operatorname{Gram}_{\mathrm{L}^{2}}\left(\Lambda_{c}^{+}\left(\begin{array}{c}
\Phi \\
0
\end{array}\right)\right) A=\operatorname{Gram}_{\mathrm{L}^{2}} \Psi_{\max }^{+}=\mathbb{1}_{\mathrm{N}} .
$$

Using the $U(N)$ invariance of $D\left(V_{c}\right)$ and $\mathcal{E}_{c}$, and the polar decomposition of square matrices, one can assume, without restricting the generality, that $A=A^{*}$ and $A$ is positive definite. Recalling that $\operatorname{Gram}_{\mathrm{L}^{2}} \Phi=\mathbb{I}_{\mathrm{N}}$, we see, 
from (31), that $\operatorname{Gram}_{\mathrm{L}^{2}}\left(\Lambda_{\mathrm{C}}^{+}\left(\begin{array}{l}\Phi \\ 0\end{array}\right)\right)=\mathbf{I}_{\mathrm{N}}+\mathrm{o}(1)$. So (37) implies $A^{2}=\mathbb{I}_{\mathrm{N}}+\mathrm{o}(1)$, hence $A=\mathbb{I}_{\mathrm{N}}+\mathrm{o}(1)$. Combining this with (31), we get

$$
\left\|\psi_{k, \max }^{+}-\left(\begin{array}{c}
\varphi_{k} \\
0
\end{array}\right)\right\|_{H^{1}}=o(1)_{c \rightarrow+\infty} .
$$

Now, since $\psi_{k, \text { max }}^{+} \in E_{c}^{+}, H_{c} \psi_{k, \text { max }}^{+}=\sqrt{c^{4}-c^{2} \Delta} \psi_{k, \text { max }}^{+}$. But

$$
\sqrt{c^{4}-c^{2} \Delta} \leq c^{2}-\frac{\Delta}{2} .
$$

This inequality is easily obtained in the Fourier domain: it follows from $\sqrt{1+x} \leq 1+\frac{x}{2}(\forall x \geq 0)$. So we get

$$
\sum_{k=1}^{N}\left(H_{c} \psi_{k, \text { max }}^{+}, \psi_{k, \text { max }}^{+}\right)_{L^{2}} \leq N c^{2}+\frac{1}{2} \sum_{k=1}^{N}\left\|\nabla \psi_{k, \text { max }}^{+}\right\|_{L^{2}}^{2} .
$$

Combining this with (31), we find

$$
\mathcal{E}_{c}\left(\Psi_{\max }^{+}\right) \leq N c^{2}+\mathcal{E}_{H F}(\Phi)+\circ(1)_{c \rightarrow+\infty} .
$$

Finally, (35) and (38) imply

$$
\begin{aligned}
E_{1, D F}^{c} & \leq \mathcal{E}_{c}\left(\Psi_{\text {max }}^{+}\right)+\circ(1)_{c \rightarrow+\infty} \\
& \leq N c^{2}+\mathcal{E}_{H F}(\Phi)+\circ(1)_{c \rightarrow+\infty}
\end{aligned}
$$

Since $\Phi$ is arbitrary, (39) implies (29). The formulas (15), (16) of Theorem 5 are thus proved.

We now check the last assertion about the $\varepsilon_{k}^{c, 1}, k=1, \ldots, N$, being the smallest eigenvalues of the operator $\bar{H}_{c, \Psi^{c, 1}}$ for $c$ large. By Corollary 4 , we can translate this statement in the language of sequences. We take a sequence $c_{n} \rightarrow+\infty$ such that $\left\{\Psi^{c_{n}, 1}\right\}_{n}$ converges in $\left(H^{1}\left(\mathbb{R}^{3}, \mathbb{C}^{4}\right)\right)^{N}$ to some $\left(\begin{array}{c}\bar{\Phi}^{1} \\ 0\end{array}\right)$, for $n$ large enough. Let $\bar{H}_{n}:=\bar{H}_{c_{n}, \Psi^{c_{n}, 1}}$ and $\mathcal{H}_{\infty}:=\mathcal{H}_{\bar{\Phi}^{1}}$. We have $\bar{H}_{n} \psi_{k}^{c_{n}, 1}=\varepsilon_{k}^{n} \psi_{k}^{c_{n}, 1}$ and $\mathcal{H}_{\infty} \bar{\varphi}_{k}^{1}=\bar{\lambda}_{k} \bar{\varphi}_{k}^{1}$, with

$0<\varepsilon_{1}^{n} \leq \ldots \leq \varepsilon_{N}^{n}<\left(c_{n}\right)^{2}, \quad \bar{\lambda}_{1} \leq \ldots \leq \bar{\lambda}_{N}<0, \quad \bar{\lambda}_{k}=\lim _{n \rightarrow+\infty}\left(\varepsilon_{k}^{n}-\left(c_{n}\right)^{2}\right)$.

Let us denote $e_{1}^{n} \leq \ldots \leq e_{i}^{n} \leq \cdots$ the sequence of eigenvalues of $\bar{H}_{n}$, in the interval $\left(0, c_{n}^{2}\right)$, counted with multiplicity. Similarly, we shall denote $\bar{\nu}_{1} \leq \ldots \leq \bar{\nu}_{i} \leq \cdots$ the sequence of eigenvalues of $\mathcal{H}_{\infty}$ in the interval 
$(-\infty, 0)$, counted with multiplicity. Let $z \in \mathbb{C} \backslash \sigma\left(\mathcal{H}_{\infty}\right)$. Then for $n$ large enough, $z+\left(c_{n}\right)^{2} \in \mathbb{C} \backslash \sigma\left(\bar{H}_{n}\right)$, and the resolvent

$$
R_{n}\left(z+\left(c_{n}\right)^{2}\right):=\left(\left(z+\left(c_{n}\right)^{2}\right) I-\bar{H}_{n}\right)^{-1}
$$

converges in norm towards the operator $L(z):\left(\begin{array}{l}\varphi \\ \chi\end{array}\right) \rightarrow\left(\begin{array}{c}\bar{R}(z) \varphi \\ 0\end{array}\right)$, where $\bar{R}(z):=\left(z I-\mathcal{H}_{\infty}\right)^{-1}$ is the resolvent of $\mathcal{H}_{\infty}$. So, by the standard spectral theory, $\lim _{n \rightarrow+\infty}\left(e_{i}^{n}-\left(c_{n}\right)^{2}\right)=\bar{\nu}_{i}$ for all $i \geq 1$.

We know that $\bar{\Phi}^{1}$ is a ground state of the Hartree-Fock model. So a result proved in [1] tells us that $\bar{\nu}_{k}=\bar{\lambda}_{k}$ for all $1 \leq k \leq N$, and $\bar{\nu}_{N+1}>\bar{\lambda}_{N}$. But $\left(\varepsilon_{N}^{n}-\left(c_{n}\right)^{2}\right)$ converges to $\bar{\lambda}_{N}$, and $\left(e_{N+1}^{n}-\left(c_{n}\right)^{2}\right)$ converges to $\bar{\nu}_{N+1}$, as $n$ goes to infinity. So, for $n$ large enough, $e_{N+1}^{n}>\varepsilon_{N}^{n}$, hence $\varepsilon_{k}^{n}=e_{k}^{n}$ for all $1 \leq k \leq N$. This ends the proof of Theorem 5 .

\section{Proof of Theorem 6}

In this section, both $\Phi$ and $\Psi$ will denote $N$-uples of 4 -spinors (functions from $\mathbb{R}^{3}$ into $\left.\mathbb{C}^{4}\right)$. As already noted above, in [6] the solution $\Psi^{c, 1}$ was obtained by a complicated min-max argument. For $c$ large, we will show that this argument can be simplified.

First of all, we introduce the notion of projector " $\varepsilon$-close to $\Lambda_{c}^{+}$", where $\Lambda_{c}^{+}=\frac{1}{2}\left|H_{c}\right|^{-1}\left(H_{c}+\left|H_{c}\right|\right)$ is the positive free-energy projector.

Definition 8 : Let $P^{+}$be an orthogonal projector in $L^{2}\left(\mathbb{R}^{3}, \mathbb{C}^{4}\right)$, whose restriction to $H^{\frac{1}{2}}\left(\mathbb{R}^{3}, \mathbb{C}^{4}\right)$ is a bounded operator on $H^{\frac{1}{2}}\left(\mathbb{R}^{3}, \mathbb{C}^{4}\right)$.

Given $\varepsilon>0, P^{+}$is $\varepsilon$-close to $\Lambda_{c}^{+}$if and only if, for all $\psi \in H^{\frac{1}{2}}\left(\mathbb{R}^{3}, \mathbb{C}^{4}\right)$,

$$
\left\|\left(-c^{2} \Delta+c^{4}\right)^{\frac{1}{4}}\left(P^{+}-\Lambda_{c}^{+}\right) \psi\right\|_{L^{2}\left(\mathbb{R}^{3}, \mathbb{C}^{4}\right)} \leq \varepsilon\left\|\left(-c^{2} \Delta+c^{4}\right)^{\frac{1}{4}} \psi\right\|_{L^{2}\left(\mathbb{R}^{3}, \mathbb{C}^{4}\right)} .
$$

An obvious example of projector $\varepsilon$-close to $\Lambda_{c}^{+}$is $\Lambda_{c}^{+}$itself. More interesting examples will be given below. Let us now give a min-max principle associated to $P^{+}$:

Lemma 9 . Fix $N, Z$ with $N<Z+1$. Take $c>0$ large enough, and $P^{+}$ a projector $\varepsilon$-close to $\Lambda_{c}^{+}$, for $\varepsilon>0$ small enough. Let $P^{-}=\mathbb{I}_{\mathrm{L}^{2}}-\mathrm{P}^{+}$, and define

$$
E\left(P^{+}\right):=\inf _{\substack{\Phi^{+} \in\left(P^{+} H^{\frac{1}{2}}\right)^{N} \\ \operatorname{Gram}_{\mathrm{L}^{2}} \Phi^{+}=\mathbf{I}_{\mathrm{N}}}} \sup _{\substack{\Psi \in\left(P^{-} H^{\frac{1}{2}} \oplus \operatorname{Span}\left(\Phi^{+}\right)\right)^{N} \\ \operatorname{Gram}_{\mathrm{L}^{2}} \Psi=\mathbf{I}_{\mathrm{N}}}} \mathcal{E}_{c}(\Psi) .
$$


Then $E\left(P^{+}\right)$does not depend on $P^{+}$and $\mathcal{E}_{c}\left(\Psi^{c, 1}\right) \leq E\left(P^{+}\right)$.

Remark. In the case $N=1, \mathcal{E}_{c}$ is the quadratic form $(\psi, H \psi)_{L^{2}}$ associated to the operator $H=H_{c}-Z \mu * \frac{1}{x \mid}$. Then $E\left(\Lambda_{c}^{+}\right)$coincides with the min-max level $\lambda_{1}(V)$ defined in [4], for $V=-Z \mu * \frac{1}{|x|}$. By Theorem 3.1 of [4], if $c>\frac{\pi / 2+2 / \pi}{2}$, then $\lambda_{1}(V)$ is the first positive eigenvalue of $H$.

\section{Proof of Lemma 9.}

The idea behind this lemma is inspired by [2]. Note that, under our assumptions, $E\left(P^{+}\right)<N c^{2}(1+K \varepsilon)$ for some $K>0$ independent of $c$ and $\varepsilon$. This follows from arguments similar to those used in the proof of Lemma 5.3 of [6]. In [6] the free energy projectors $\Lambda_{c}^{ \pm}$were used. With these projectors, it was seen that $E\left(\Lambda_{c}^{+}\right)<N c^{2}$ (thanks to a careful choice of $\left.\Phi^{+}\right)$. When $P^{+}$is $\varepsilon$-close to $\Lambda_{c}^{+}$, we then get $E\left(P^{+}\right)<N c^{2}(1+K \varepsilon)$.

To continue the proof of the lemma we perform a change of physical units. In mathematical language, this change corresponds to a dilation in space by the factor $c$, and to dividing the energies by $c^{2}$. Let $\left(d_{c} \varphi\right)(x)=c^{3 / 2} \varphi(c x)$ and

$$
\begin{aligned}
\widetilde{\mathcal{E}}_{c}(\Phi):= & \frac{1}{c^{2}} \mathcal{E}_{c}\left(d_{c} \Phi\right) \\
= & \sum_{k=1}^{N} \int_{\mathbb{R}^{3}}\left(\varphi_{k},(-i \alpha \cdot \nabla+\beta) \varphi_{k}\right)-\frac{Z}{c}\left(\widetilde{\mu} * \frac{1}{|x|}\right)\left|\varphi_{k}\right|^{2} \\
& +\frac{1}{2 c} \iint_{\mathbb{R}^{3} \times \mathbb{R}^{3}} \frac{\rho_{\Phi}(x) \rho_{\Phi}(y)-\left\|R_{\Phi}(x, y)\right\|^{2}}{|x-y|} d^{3} x d^{3} y
\end{aligned}
$$

where $\tilde{\mu}(E)=\mu\left(c^{-1} E\right)$ for any Borel subset $E$ of $\mathbb{R}^{3}$.

The interest of this rescaled energy $\widetilde{\mathcal{E}}_{c}$ is that for $c$ large and $\operatorname{Gram}_{\mathrm{L}^{2}} \Psi \leq \mathbf{I}_{\mathrm{N}}$, we have

$$
\widetilde{\mathcal{E}}_{c}(\Psi)=\sum_{k=1}^{N} \int_{\mathbb{R}^{3}}\left(\psi_{k},(-i \alpha \nabla+\beta) \psi_{k}\right)+O\left(\frac{1}{c}\|\Psi\|_{\left(H^{1 / 2}\right)^{N}}^{2}\right) .
$$

Let us denote $\widetilde{P}^{ \pm}:=d_{c^{-1}} \circ P^{ \pm} \circ d_{c}, \widetilde{\Lambda}^{ \pm}:=d_{c^{-1}} \circ \Lambda_{c}^{ \pm} \circ d_{c}=\chi_{\mathbb{R}_{ \pm}}(-i \alpha . \nabla+\beta)$. Note that $\widetilde{\Lambda}^{ \pm}$does not depend on $c$. Now, $P^{+}$is $\varepsilon$-close to $\Lambda_{c}^{+}$if and only if

$$
\left\{\begin{aligned}
\|(-\Delta+1)^{\frac{1}{4}} & \left(\widetilde{P}^{+}-\widetilde{\Lambda}^{+}\right) \psi \|_{L^{2}\left(\mathbb{R}^{3}, \mathbb{C}^{4}\right)} \\
& \leq \varepsilon\left\|(-\Delta+1)^{\frac{1}{4}} \psi\right\|_{L^{2}\left(\mathbb{R}^{3}, \mathbb{C}^{4}\right)}, \quad \forall \psi \in H^{\frac{1}{2}}\left(\mathbb{R}^{3}, \mathbb{C}^{4}\right) .
\end{aligned}\right.
$$


We denote $\Phi \bullet A$ the right action of an $N \times N$ matrix $A=\left(a_{k l}\right)_{1 \leq k, l \leq N}$ on an $N$-uple $\Phi=\left(\varphi_{1}, \ldots, \varphi_{N}\right) \in\left(L^{2}\left(\mathbb{R}^{3}, \mathbb{C}^{4}\right)\right)^{N}$. More precisely,

$$
(\Phi \bullet A):=\left(\sum_{k=1}^{N} a_{k 1} \varphi_{k}, \ldots, \sum_{k=1}^{N} a_{k N} \varphi_{k}\right) .
$$

Given $\Phi^{+}=\left(\varphi_{1}^{+}, \ldots, \varphi_{N}^{+}\right) \in\left(\widetilde{P}^{+} H^{1 / 2}\right)^{N}$ such that $\operatorname{Gram}_{\mathrm{L}^{2}} \Phi^{+}=\mathbf{I}_{\mathrm{N}}$, and $\Phi^{-} \in\left(\widetilde{P}^{-} H^{1 / 2}\right)^{N}$, we define

$$
\begin{aligned}
g_{\Phi^{+}}\left(\Phi^{-}\right): & :=\left(\Phi^{+}+\Phi^{-}\right) \bullet\left[\operatorname{Gram}_{\mathrm{L}^{2}}\left(\Phi^{+}+\Phi^{-}\right)\right]_{1}^{-\frac{1}{2}} \\
& =\left(\Phi^{+}+\Phi^{-}\right) \bullet\left[\mathbb{I}_{\mathrm{N}}+\operatorname{Gram}_{\mathrm{L}^{2}} \Phi^{-}\right]^{-\frac{1}{2}}
\end{aligned}
$$

We obtain a smooth map $g_{\Phi^{+}}$, from $\left(\widetilde{P}^{-} H^{\frac{1}{2}}\right)^{N}$ to

$$
\Sigma_{\Phi^{+}}:=\left\{\Psi \in\left(\widetilde{P}^{-} H^{\frac{1}{2}} \oplus \operatorname{Span}\left(\varphi_{1}^{+}, \ldots, \varphi_{N}^{+}\right)\right)^{N} / \operatorname{Gram}_{\mathrm{L}^{2}} \Psi=\mathbb{I}_{\mathrm{N}}\right\} .
$$

In fact, the values of $g_{\Phi^{+}}$lie in the following subset of $\Sigma_{\Phi^{+}}$:

$$
\Sigma_{\Phi^{+}}^{\prime}:=\left\{\Psi \in \Sigma_{\Phi^{+}} / \operatorname{Gram}_{\mathrm{L}^{2}}\left(\widetilde{P}^{+} \Psi\right)>0\right\} .
$$

Now, take an arbitrary $\Psi \in \Sigma_{\Phi^{+}}^{\prime}$. Then there is an invertible $N \times N$ matrix $B$ such that $\widetilde{P}^{+} \Psi=\Phi^{+} \bullet B$. So we may write

$$
\Psi \bullet B^{-1}=\Phi^{+}+\widetilde{P}^{-} \Psi \bullet B^{-1} .
$$

As a consequence,

$$
g_{\Phi^{+}}\left(\widetilde{P}^{-} \Psi \bullet B^{-1}\right)=\left(\Psi \bullet B^{-1}\right) \bullet\left[\operatorname{Gram}_{\mathrm{L}^{2}}\left(\Psi \bullet B^{-1}\right)\right]^{-\frac{1}{2}} .
$$

One easily computes

$$
\operatorname{Gram}_{\mathrm{L}^{2}}\left(\Psi \bullet B^{-1}\right)=\left(B^{*}\right)^{-1}\left(\operatorname{Gram}_{\mathrm{L}^{2}} \Psi\right) B^{-1}=\left(B B^{*}\right)^{-1} .
$$

Hence

$$
g_{\Phi^{+}}\left(\widetilde{P}^{-} \Psi \bullet B^{-1}\right)=\left(\Psi \bullet B^{-1}\right) \bullet\left(B B^{*}\right)^{1 / 2}=\Psi \bullet\left(B^{-1}\left(B B^{*}\right)^{1 / 2}\right),
$$

and finally

$$
\Psi=g_{\Phi^{+}}\left(\widetilde{P}^{-} \Psi \bullet B^{-1}\right) \bullet U
$$


where $U:=\left(B B^{*}\right)^{-1 / 2} B \in \mathcal{U}(N)$ is the unitary matrix appearing in the polar decomposition of $B$. So we have proved that

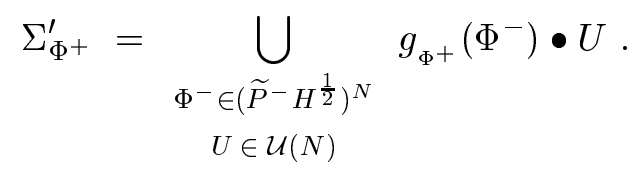

Now, $\mathcal{E}_{c}$ is invariant under the $\mathcal{U}(N)$ action " $\bullet "$, and $\Sigma_{\Phi^{+}}^{\prime}$ is dense in $\Sigma_{\Phi^{+}}$for the norm of $\left(H^{1 / 2}\left(\mathbb{R}^{3}, \mathbb{C}^{4}\right)\right)^{N}$. Hence

$$
\sup _{\substack{\Psi \in\left(\widetilde{P}^{-} H^{\frac{1}{2}} \oplus \operatorname{Span}\left(\Phi^{+}\right)\right)^{N} \\ \operatorname{Gram}_{\mathrm{L}^{2}} \Psi=\mathbb{I}_{\mathrm{N}}}} \widetilde{\mathcal{E}}_{c}(\Psi)=\sup _{\Phi^{-} \in\left(\widetilde{P}^{-} H^{\frac{1}{2}}\right)^{N}} \widetilde{\mathcal{E}}_{c}\left(g_{\Phi^{+}}\left(\Phi^{-}\right)\right) .
$$

We now prove Lemma 9 in three steps.

Step 1. Let $\Phi^{+} \in\left(\widetilde{P}^{+} H^{1 / 2}\right)^{N}$ be such that $\operatorname{Gram}_{\mathrm{L}^{2}} \Phi^{+}=\mathbf{I}_{\mathrm{N}}$ and such that $\widetilde{\mathcal{E}}_{c}\left(\Phi^{+}\right) \leq N+\delta$, for some $\delta>0$ small. For $\varepsilon$ small and $c$ large, there is a unique $\Phi^{-} \in\left(\widetilde{P}^{-} H^{1 / 2}\right)^{N}$ maximizing $\widetilde{\mathcal{E}}_{c} \circ g_{\Phi^{+}}$and lying in a small neighborhood of 0 . If we denote $k\left(\Phi^{+}\right)$this maximizer, the map $k$ is smooth from

$$
\mathcal{S}_{\delta}^{+}=\left\{\Phi^{+} \in\left(\widetilde{P}^{+} H^{1 / 2}\right)^{N} / \operatorname{Gram}_{\mathrm{L}^{2}} \Phi^{+}=\mathbb{I}_{\mathrm{N}}, \widetilde{\mathcal{E}}_{\mathrm{c}}\left(\Phi^{+}\right) \leq \mathrm{N}+\delta\right\}
$$

to $\left(\widetilde{P}^{-} H^{1 / 2}\right)^{N}$, and equivariant for the $\mathcal{U}(N)$ action.

Proof of Step 1. Take $r>0$. For $\varepsilon, \delta$ small and $c$ large, if $\Phi^{+} \in \mathcal{S}_{\delta}^{+}$, $\Phi^{-} \in\left(\widetilde{P}^{-} H^{1 / 2}\right)^{N}$, and $\left\|\Phi^{-}\right\|_{H^{1 / 2}}$ is not smaller than $r$, then

$$
\widetilde{\mathcal{E}}_{c}\left(g_{\Phi^{+}}\left(\Phi^{-}\right)\right)<N-\delta,
$$

by (41). On the other hand, for $c$ large enough, using (41) once again, one has

$$
\widetilde{\mathcal{E}}_{c}\left(g_{\Phi^{+}}(0)\right)=\widetilde{\mathcal{E}}_{c}\left(\Phi^{+}\right) \geq N-\frac{\delta}{2} .
$$

So, if we define $\mathcal{V}_{r}:=\left\{\Phi^{-} \in\left(\widetilde{P}^{-} H^{1 / 2}\right)^{N} /\left\|\Phi^{-}\right\|_{H^{1 / 2}} \leq r\right\}$, no maximizer of $\widetilde{\mathcal{E}}_{c} \circ g_{\Phi+}$ can be outside $\mathcal{V}_{r}$. Moreover, choosing $r$ small, and then taking $c$ large and $\varepsilon$ small, the map

$$
\Phi^{-} \in \mathcal{V}_{r} \longmapsto \widetilde{\mathcal{E}}_{c} \circ g_{\Phi^{+}}\left(\Phi^{-}\right)
$$


is strictly concave. Indeed, its second derivative at $\Phi^{-} \in \mathcal{V}_{r}$ is very close in norm to the negative form

$\Psi^{-} \in\left(\widetilde{P}^{-} H^{1 / 2}\right)^{N} \longmapsto-2 \sum_{i=1}^{N}\left\|\psi_{i}^{-}\right\|_{H^{1 / 2}}^{2}-2 \sum_{1 \leq i, j \leq N}\left(\varphi_{j}^{+}, \varphi_{i}^{+}\right)_{H^{1 / 2}}\left(\psi_{i}^{-}, \psi_{j}^{-}\right)_{L^{2}}$.

Step 1 immediately follows from these facts.

Step 2. The min-max level $E\left(P^{+}\right)$does not depend on $P^{+}$.

Proof of Step 2. Take two projectors $P_{1}^{+}, P_{2}^{+}$, both $\varepsilon$-close to $\Lambda_{c}^{+}$. For $i=1,2$, and $\Phi_{i}^{+} \in\left(\widetilde{P}_{i}^{+} H^{1 / 2}\right)^{N}$, with $\operatorname{Gram}_{\mathrm{L}^{2}} \Phi_{i}^{+}=\mathbb{I}_{\mathrm{N}}$ and $\widetilde{\mathcal{E}}_{c}\left(\Phi_{i}^{+}\right) \leq N+\delta$, let

$$
\begin{gathered}
J^{i}\left(\Phi_{i}^{+}\right):=\max _{\begin{array}{c}
\Phi^{-} \in\left(\tilde{P}_{i}^{-} H^{1 / 2}\right)^{N} \\
\operatorname{Gram}_{\mathrm{L}^{2}} \Phi^{-}=\mathbf{I}_{\mathrm{N}}
\end{array}} \widetilde{\mathcal{E}}_{c}\left(g_{\Phi_{i}^{+}}^{i}\left(\Phi^{-}\right)\right) \\
=\widetilde{\mathcal{E}}_{c} \circ g_{\Phi_{i}^{+}}^{i}\left(k^{i}\left(\Phi_{i}^{+}\right)\right) .
\end{gathered}
$$

Here, $g_{\Phi^{+}}^{i}$ and $k^{i}$ are the maps associated to $P_{i}^{+}$in Step 1.

By Ekeland's variational principle [5], there is a minimizing sequence $\left(\Phi_{1, n}^{+}\right)_{n \geq 0}$ for $J^{1}$, such that $\left(J^{1}\right)^{\prime}\left(\Phi_{1, n}^{+}\right) \underset{n \rightarrow+\infty}{\longrightarrow} 0$ in $\left(H^{-1 / 2}\right)^{N}$. Let $\Psi_{n}:=g_{\Phi_{1, n}^{+}}^{1}\left(k^{1}\left(\Phi_{1, n}^{+}\right)\right)$.

Then $\Psi_{n}$ is a Palais-Smale sequence for $\widetilde{\mathcal{E}}_{c}$ in the manifold

$$
\Sigma:=\left\{\Psi \in\left(H^{1 / 2}\right)^{N} / \operatorname{Gram}_{\mathrm{L}^{2}} \Psi=\mathbb{I}_{\mathrm{N}}\right\},
$$

with $\widetilde{\mathcal{E}}_{c}\left(\Psi_{n}\right) \geq N-\frac{\delta}{2}$, where $\delta>0$ is the constant of the first step. So $\operatorname{Gram}_{\mathrm{L}^{2}}\left(\widetilde{P}_{2}^{+} \Psi_{n}\right)>0$. We denote

$$
\left\{\begin{array}{l}
\Phi_{2, n}^{+}:=\widetilde{P}_{2}^{+} \Psi_{n} \bullet\left[\operatorname{Gram}_{\mathrm{L}^{2}}\left(\widetilde{P}_{2}^{+} \Psi_{n}\right)\right]^{-\frac{1}{2}} \\
\Phi_{2, n}^{-}:=\widetilde{P}_{2}^{-} \Psi_{n} \bullet\left[\operatorname{Gram}_{\mathrm{L}^{2}}\left(\widetilde{P}_{2}^{+} \Psi_{n}\right)\right]^{-\frac{1}{2}}
\end{array}\right.
$$

One easily checks that $\Psi_{n}=g_{\Phi_{2, n}^{+}}^{2}\left(\Phi_{2, n}^{-}\right)$. Since $\widetilde{\mathcal{E}}_{c}\left(\Psi_{n}\right) \geq N-\frac{\delta}{2}$, we have $\left\|\Phi_{2, n}^{-}\right\|_{H^{1 / 2}} \leq r$, where $r>0$ is the same as in the proof of step 1. Since $\Psi_{n}$ is a Palais-Smale sequence for $\widetilde{\mathcal{E}}_{c}$, the derivative of $\widetilde{\mathcal{E}}_{c} \circ g_{\Phi_{2, n}^{+}}^{2}$ at the point 
$\Phi_{2, n}^{-}$converges to 0 as $n$ goes to infinity. So, by the concavity properties of $\widetilde{\mathcal{E}}_{c} \circ g_{\Phi_{2, n}^{+}}^{2}$ in the domain

$$
\mathcal{V}_{2, r}:=\left\{\Phi^{-} \in\left(\widetilde{P}_{2}^{-} H^{1 / 2}\right)^{N} /\left\|\Phi^{-}\right\|_{H^{1 / 2}} \leq r\right\}
$$

(see the proof of step 1), we get

$\left\|\Phi_{2, n}^{-}-k^{2}\left(\Phi_{2, n}^{+}\right)\right\|_{H^{1 / 2}} \quad \underset{n \rightarrow+\infty}{\longrightarrow} \quad 0 \quad$ and $\quad \widetilde{\mathcal{E}}_{c}\left(\Psi_{n}\right)-J^{2}\left(\Phi_{2, n}^{+}\right) \underset{n \rightarrow+\infty}{\longrightarrow} 0$.

As a consequence,

$$
E\left(P_{1}^{+}\right)=\inf _{\substack{\Phi_{1}^{+} \in\left(\tilde{P}_{1}^{+} H^{1 / 2}\right)^{N} \\ \operatorname{Gram}_{\mathrm{L}^{2}} \Phi_{1}^{+}=\mathbf{I}_{\mathrm{N}}}} J^{1}\left(\Phi_{1}^{+}\right) \geq \inf _{\substack{\Phi_{2}^{+} \in\left(\tilde{P}_{2}^{+} H^{1 / 2}\right)^{N} \\ \operatorname{Gram}_{\mathrm{L}^{2}} \Phi_{2}^{+}=\mathbb{I}_{\mathrm{N}}}} J^{2}\left(\Phi_{2}^{+}\right)=E\left(P_{2}^{+}\right) .
$$

Since 1,2 play symmetric roles in the above arguments, we conclude that $E\left(P^{+}\right)$does not depend on $P^{+}$, for $c$ large enough and $\varepsilon$ small enough.

Step 3. $\mathcal{E}_{c}\left(\Psi^{c, 1}\right) \leq E\left(\Lambda_{c}^{+}\right)$, where $\Psi^{c, 1}$ is the first solution of $(D-F)$ found in $[E-S]$.

\section{Proof of Step 3.}

For $c$ large enough, if $\Psi^{-} \in \Lambda_{c}^{-} H^{1 / 2}$ satisfies $\operatorname{Gram}_{\mathrm{L}^{2}} \Psi^{-} \leq \mathbf{I}_{\mathrm{N}}$, it follows from Hardy's inequality that the map $\Psi^{+} \rightarrow \mathcal{E}_{c}\left(\Psi^{+}+\Psi^{-}\right)$is strictly convex on

$$
W\left(\Psi^{-}\right):=\left\{\Psi^{+} \in\left(\Lambda_{c}^{+} H^{1 / 2}\right)^{N} ; \operatorname{Gram}_{\mathrm{L}^{2}}\left(\Psi^{+}+\Psi^{-}\right) \leq \mathbb{I}_{\mathrm{N}}\right\} .
$$

As a consequence, for an arbitrary $N$-dimensional subspace $V$ of $\Lambda_{c}^{+} H^{1 / 2}$, $S_{V}\left(\Psi^{-}\right):=\sup _{\Psi^{+} \in W\left(\Psi^{-}\right) \cap V^{N}} \mathcal{E}_{c}\left(\Psi^{+}+\Psi^{-}\right)$is achieved by an extremal point $\Psi_{\text {max }}^{+}$of the convex set $W\left(\Psi^{-}\right) \cap V^{N}$. Being extremal, $\Psi_{\max }^{+}$must satisfy the contraints $\operatorname{Gram}_{\mathrm{L}^{2}}\left(\Psi_{\text {max }}^{+}+\Psi^{-}\right)=\mathbb{I}_{\mathrm{N}}$.

So we have

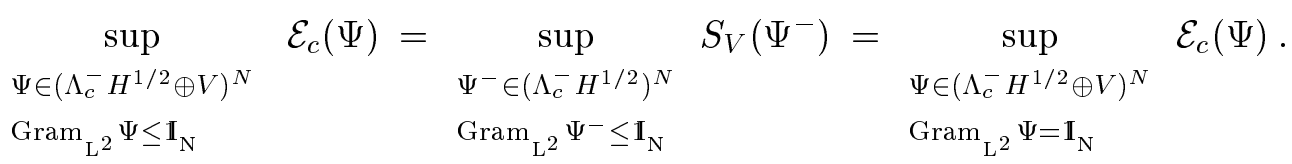


By proposition 2,

$$
\mathcal{E}_{c}\left(\Psi^{c, 1}\right) \leq \sup _{\substack{\Psi \in\left(\Lambda_{c}^{-} H^{1 / 2} \oplus V\right)^{N} \\ \operatorname{Gram}_{\mathrm{L}^{2}} \Psi \leq \mathbf{I}_{\mathrm{N}}}} \mathcal{E}_{c}(\Psi)
$$

Finally we get, for $c$ large,

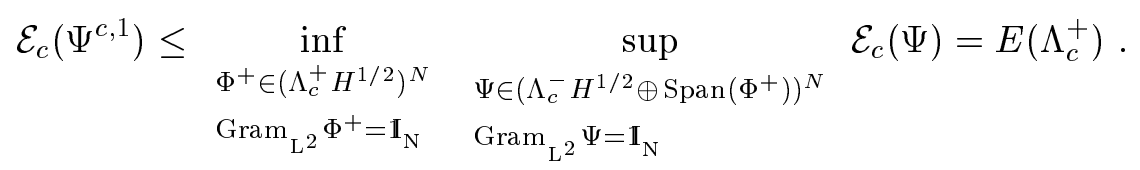

(The correspondence between $\Phi^{+}$and $V$ is $V=\operatorname{Span}\left(\Phi^{+}\right)$). This ends the proof of Step 3 and of Lemma 9.

Thanks to Lemma 9, we are able to write the following inequalities for $c$ large, and $P^{+} \varepsilon$-close to $\Lambda_{c}^{+}, \varepsilon$ small :

$$
\begin{aligned}
E\left(P^{+}\right)=E\left(\Lambda_{c}^{+}\right) & \geq \mathcal{E}_{c}\left(\Psi^{c, 1}\right) \\
& \geq \inf _{\begin{array}{l}
\Psi \text { solution of }\left(D F_{c}\right) \\
\Lambda_{\Psi}^{-} \Psi=0
\end{array}} \mathcal{E}_{c}(\Psi) \\
& \geq\left\{\begin{array}{l}
\Psi \in\left(H^{1 / 2}\right)^{N} \\
\operatorname{Gram}_{\mathrm{L}^{2}} \Psi=\mathbf{I}_{\mathrm{N}} \\
\Lambda_{\Psi}^{-} \Psi=0
\end{array}\right.
\end{aligned}
$$

As announced before, we now give some important examples of projectors $\varepsilon$-close to $\Lambda_{c}^{+}$:

Lemma 10. Fix $N, Z$, and take c large enough. Then, for any $\Phi \in$ $\left(H^{1 / 2}\right)^{N}$, with $\operatorname{Gram}_{\mathrm{L}^{2}} \Phi \leq \mathbf{I}_{\mathrm{N}}$, the projector $\Lambda_{\Phi}^{+}=\chi_{(0,+\infty)}\left(\bar{H}_{c, \Phi}\right)$ is $\varepsilon$ close to $\Lambda_{c}^{+}$.

Proof of Lemma 10. We adapt a method of Griesemer, Lewis, Siedentop [7] to the Hamiltonian $\bar{H}_{c, \Phi}$. Once again, it is more convenient to work in a system of units such that $\bar{H}_{c, \Phi}$ becomes

$$
\begin{aligned}
\widetilde{H}_{c, \tilde{\Phi}}: \psi \mapsto d_{c^{-1}} \circ \bar{H}_{c, \Phi} \circ d_{c}(\psi)=(-i \alpha \cdot \nabla+\beta) \psi- & \frac{Z}{c}\left(\tilde{\mu} * \frac{1}{|x|}\right) \psi \\
& +\frac{1}{c}\left(\rho_{\tilde{\Phi}} * \frac{1}{|x|}\right) \psi-\frac{1}{c} \int_{\mathbb{R}^{3}} R_{\tilde{\Phi}}(x, y) \frac{\psi(y)}{|x-y|} d y
\end{aligned}
$$


with $\widetilde{\mu}(E)=\mu\left(c^{-1} E\right), \widetilde{\Phi}(x)=c^{-3 / 2} \Phi\left(c^{-1} x\right)$.

Denoting $H_{1}:=-i \alpha \cdot \nabla+\beta, \widetilde{\Lambda}_{\tilde{\Phi}}^{+}:=\chi_{(0, \infty)}\left(\widetilde{H}_{c, \tilde{\Phi}}\right), \widetilde{\Lambda}^{+}:=\chi_{(0, \infty)}\left(H_{1}\right)$, $K_{\tilde{\Phi}}:=c\left(\widetilde{H}_{c, \tilde{\Phi}}-H_{1}\right)$, we find, as in the proof of Lemma 1 of [7],

$$
\left(\widetilde{\Lambda}_{\tilde{\Phi}}^{+}-\widetilde{\Lambda}^{+}\right) \psi=\frac{1}{\pi c} \int_{0}^{+\infty} d z\left[H_{1}^{2}+z^{2}\right]^{-1}\left(H_{1} K_{\tilde{\Phi}} \widetilde{H}_{c, \tilde{\Phi}}-z^{2} K_{\tilde{\Phi}}\right)\left[\left(\widetilde{H}_{c, \tilde{\Phi}}\right)^{2}+z^{2}\right]^{-1} \psi,
$$

and for any $\chi \in L^{2}\left(\mathbb{R}^{3}, \mathbb{C}^{4}\right)$, following [7] (proof of Lemma 3), we get

$$
\left(\chi,(-\Delta+1)^{1 / 4}\left(\widetilde{\Lambda}_{\tilde{\Phi}}^{+}-\widetilde{\Lambda}^{+}\right) \psi\right)_{L^{2}} \leq \frac{M}{c}\|\chi\|_{L^{2}}\left\|(-\Delta+1)^{1 / 4} \psi\right\|_{L^{2}}
$$

for $c$ large enough ( $M$ is a constant independent of $c$ ). As a consequence, if $c$ is large enough and bigger than $\frac{M}{\varepsilon}$, then $\Lambda_{\Phi}^{+}$is $\varepsilon$-close to $\Lambda_{c}^{+}$. This ends the proof of Lemma 10.

Now, to end the proof of Theorem 6 , we just need the following result :

Lemma 11 . Fix $N, Z$ and take $c>0$ large enough. If $\Phi \in\left(H^{1 / 2}\right)^{N}$, $\operatorname{Gram}_{\mathrm{L}^{2}} \Phi=\mathbf{I}_{\mathrm{N}}, \Lambda_{\Phi}^{-} \Phi=0$ and $\mathcal{E}_{c}(\Phi) \leq N c^{2}$, then

$$
\mathcal{E}_{c}(\Phi)=\max \left\{\mathcal{E}_{c}(\Psi) ; \Psi \in\left[\Lambda_{\Phi}^{-} H^{1 / 2} \oplus \operatorname{Span}(\Phi)\right]^{N}, \operatorname{Gram}_{\mathrm{L}^{2}} \Psi=\mathbf{I}_{\mathrm{N}}\right\}
$$

Proof of Lemma 11. If $\Lambda_{\Phi}^{-} \Phi=0$ and $\operatorname{Gram}_{\mathrm{L}^{2}} \Phi=\mathbb{I}_{\mathrm{N}}$, then 0 is a critical point of the map

$$
\Psi^{-} \in\left(\Lambda_{\Phi}^{-} H^{1 / 2}\right)^{N} \longmapsto \mathcal{E}_{c}\left(g_{\Phi}\left(\Psi^{-}\right)\right)
$$

with $g_{\Phi}\left(\Psi^{-}\right)=\left(\Phi+\Psi^{-}\right) \bullet\left[\mathbf{I}_{\mathrm{N}}+\operatorname{Gram}_{\mathrm{L}^{2}} \Psi^{-}\right]^{-1 / 2}$. Take $\varepsilon>0$ small. By Lemma $10, \Lambda_{\Phi}^{+}$is $\varepsilon$-close to $\Lambda_{c}^{+}$for $c$ large enough. From the proof of Lemma 9 (Step 1), there is a unique critical point of $\mathcal{E}_{c} \circ g_{\Phi}$ in a small neighborhood $\mathcal{V}_{r}$ of 0 in $\Lambda_{\Phi}^{-}\left(H^{1 / 2}\right)$ and this critical point is the unique maximizer of $\mathcal{E}_{c} \circ g_{\Phi}$ in $\Lambda_{\Phi}^{-}\left(H^{1 / 2}\right)$. So, 0 is this maximizer. This proves Lemma 11.

Let us explain why Theorem 6 is now proved. We know that, for $c$ large enough,

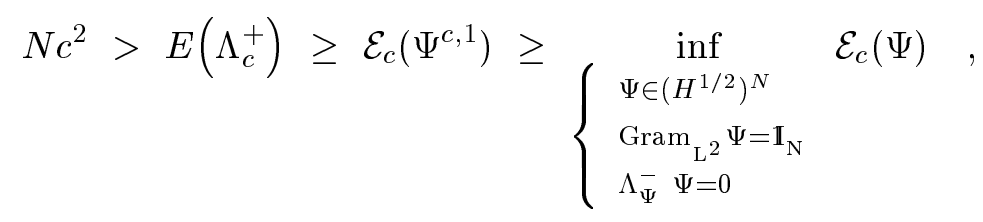


hence

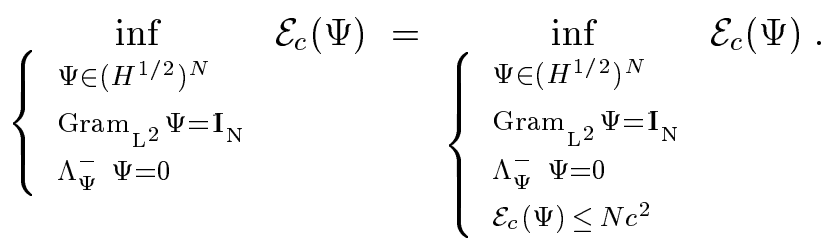

Take $\varepsilon>0$. By Lemma 10, for any $\Psi \in\left(H^{1 / 2}\right)^{N}$ with $\operatorname{Gram}_{\mathrm{L}^{2}} \Psi=\mathbf{I}_{\mathrm{N}}$, the projector $\Lambda_{\Psi}^{+}$is $\varepsilon$-close to $\Lambda_{c}^{+}$, if $c$ is large. Hence $E\left(\Lambda_{\Psi}^{+}\right)=E\left(\Lambda_{c}^{+}\right)$(by Lemma 9), if we have chosen $\varepsilon$ small enough. But if $\Psi$ also satisfies $\Lambda_{\Psi}^{-} \Psi=0$ and $\mathcal{E}_{c}(\Psi) \leq N c^{2}$, then, from Lemma 11 and from the definition of $E\left(\Lambda_{\Psi}^{+}\right)$, we have $E\left(\Lambda_{c}^{+}\right)=E\left(\Lambda_{\Psi}^{+}\right) \leq \mathcal{E}_{c}(\Psi)$. So

$$
E\left(\Lambda_{c}^{+}\right) \leq \inf _{\Psi \in\left(H^{1 / 2}\right)^{N}} \mathcal{E}_{c}(\Psi),
$$

and therefore,

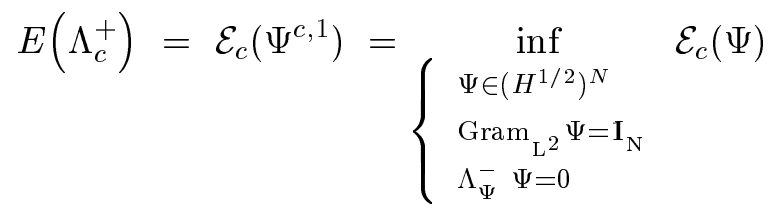

and Theorem 6 is proved.

Aknowledgements. The authors are grateful to Boris Buffoni for explaining them the work [2], and suggesting that it might be useful in the study of the Dirac-Fock functional. The proof of Lemma 9 is inspired by this paper.

\section{References}

[1] V. Bach, E.H. Lieb, M. Loss, J.P. Solovej. There are no unfilled shells in unrestricted Hartree-Fock theory. Phys. Rev. Lett. 72(19) (1994), p. 2981-2983.

[2] B. Buffoni, L. Jeanjean. Minimax characterization of solutions for a semi-linear elliptic equation with lack of compactness. Ann. Inst. H. Poincaré 10(4) (1993), p. 377-404. 
[3] V.I. Burenkov, W.D. Evans. On the evaluation of the norm of an integral operator associated with the stability of one-electron atoms. Proc. Roy. Soc. Edinburgh, sect. A 128 (5) (1998), p. 993-1005.

[4] J. Dolbeault, M.J. Esteban, E. Séré. Variational characterization for eigenvalues of Dirac operators. Cal. Var. and PDE 10 (4) (2000), p. 321-347.

[5] I. Ekeland. On the variational principle. J. Math. Anal. 47 (1974), p. 324-353.

[6] M.J. Esteban, E. Séré. Solutions for the Dirac-Fock equations for atoms and molecules. Comm. Math. Phys. 203 (1999), p. 499-530.

[7] M. Griesemer, R.T. Lewis, H. Siedentop. A minimax principle for eigenvalues in spectral gaps : Dirac operators with Coulomb potentials. Doc. Math. 4 (1999), p. 275-283.

[8] I.W. Herbst. Spectral theory of the operator $\left(p^{2}+m^{2}\right)^{1 / 2}-z e^{2} / r$. Comm. Math. Phys. 53 (1977), p. 285-294.

[9] Y.-K. Kim. Relativistic self-consistent field theory for closed-shell atoms. Phys. Rev. 154 (1967), p. 17-39.

[10] E. H. Lieb, B. Simon. The Hartree-Fock theory for Coulomb systems. Comm. Math. Phys., 53 (1977), p. 185-194.

[11] P.-L. Lions. Solutions of Hartree-Fock equations for Coulomb systems. Comm. Math. Phys. 109 (1987), p. 33-97.

[12] A. Messiah. Mécanique quantique. Dunod, 1965.

[13] E. Paturel. Solutions of the Dirac equations without projector. To appear in A.H.P.

[14] B. Thaller. The Dirac equation. Springer-Verlag, 1992.

[15] C. Tix. Strict positivity of a relativistic Hamiltonian due to Brown and Ravenhall. Bull. London Math. Soc. 30(3) (1998), p. 283-290.

[16] C. Tix. Lower bound for the ground state energy of the no-pair Hamiltonian. Phys. Lett. B 405 (1997), p. 293-296. 